\title{
Infraspinatus and Teres Minor Tendon Calcification with Severe Pain
}

\author{
Zhen Yang ${ }^{1}$, Wengiu Zhao ${ }^{2}$, Yongjun Yang ${ }^{2}$ \\ ${ }^{1}$ University of Western Ontario, 'Kingsway Xray and Ultrasound
}

Calcific tendinitis is an extremely common shoulder disorder which affects 2.7 to $10.3 \%$ of the adult population. However, clear details regarding the formation of calcific tendinitis have only been found recently, and its pathology still under debate. In this report, we present a case of calcific tendinitis affect both the infraspinatus and the teres minor tendon with both X-ray and ultrasound findings. Calcific tendinitis at the infraspinatus is rare (around $8.4 \%$ in all rotator cuff calcific tendinitis), and no separate case report on calcific tendinitis has been reported in the teres minor region in the preceding several years, therefore, the rarity of reporting this case was considered. ${ }^{4}$ We assume that additional case reports can help to further improve our understanding regarding infraspinatus and teres minor tendon calcification.

\section{Introduction}

Rotator cuff calcific tendinitis, or calcific tendinopathy, is a common shoulder disorder and a major cause of shoulder pain. It is a self-limited disorder of unclear etiology that is characterized by the formation of crystalline calcium hydroxyapatite in the rotator cuff tendon, which may be followed by the spontaneous resorption of this calcium compound and subsequent tendon healing. ${ }^{5}$ It has been reported that between $2.7 \%$ to $10 \%$ of the adult population are impacted by this disorder. Calcific tendinitis occurs primarily during late adulthood, between approximately 30 and 60 years of age, and is more frequently developing among sedentary workers than among physical laborers. ${ }^{6-8}$ Reports have indicated that among all populations, middleaged, office-working women have the highest probability of suffering from calcific tendinitis of the rotator cuff. ${ }^{9}$ The occurrence rate of calcific tendinitis at the infraspinatus among all cases of calcific tendinitis around the rotator cuff is $2.6 \%$. ${ }^{4}$ Although many case reports and meta-analyses regarding this type of disease have been published, various characteristics such as the cause of disease and effective ratio among the population for this disease have not been clearly established. 1,6-8,10-12 For example, with respect to the prevalence between two shoulders in asymptomatic groups, certain article suggest that both shoulders are equally affected, ${ }^{1}$ whereas other articles have reported that calcification exhibits a predilection for the right side. ${ }^{10,13}$ No correlation between the dominant arm and the affected shoulder has been found; the disorder occurs randomly on the both of the arms. $^{1,4,10,13,14}$ We report a case involving infraspinatus and teres minor tendon calcification and review the relevant literature.

\section{Methods}

A healthy forty-year-old man suddenly experienced the onset of severe acute pain in the left lateral shoulder, which forced him to return from a trip to obtain an urgent MSK (Musculoskeletal) ultrasound exam to exclude septic arthritis of the shoulder. The patient complained that the pain began suddenly without external physical damage. Even rest and the application of ice did not diminish the patient's pain sufficiently to allow him to perform all of his daily activities. Oral consent obtained and report was performed according to the Declaration of Helsinki principles.

A physical systemic exam was negative, with no weight loss, fever, sweats, or chills. Physical examination revealed a painful shoulder. The patient did not have any conditions that provoked calcific deposits, such as gouty arthritis, renal disease, parathyroid hormone disease, connective tissue disease, or tumoral calcinosis.

Clinical assessment revealed a severe and painful limited arc of motion, with a positive Neer impingement sign and a markedly positive O'Brien's sign. The patient exhibited severe weakness during resisted external rotation and abduction testing. His belly press and liftoff tests were normal, and he demonstrated no signs of instability.

Investigations included radiography and ultrasound. The ultrasound was performed by a 
Sonographer with more than 10 years of musculoskeletal scanning experience (Philips HD15). The shoulder examination was performed according to a routine shoulder protocol.

\section{Results}

Initial radiography of the left shoulder did not show any calcification (Figure 1), MSK ultrasound included transverse and longitudinal scanning planes for the biceps tendon, the subscapularis, the supraspinatus, the infraspinatus and teres minor tendons. The patient's hand was put on the contralateral shoulder during the infraspinatus and teres minor tendons examination. A dynamic view of the infraspinatus and teres minor tendons was obtained when the shoulder was moved into external rotation. The tendon in hyperechoic fibrillary layer. IIIdefined more hyperechoic irregular calcifications without shadowing in the infraspinatus and teres minor tendons was revealed adjacent to the posterior humeral head that were consistent with calcific infraspinatus and teres minor tendinitis. The size of calcification was measured $8.3 \times 3.1 \times 22.3 \mathrm{~mm}$. (Figures 2).

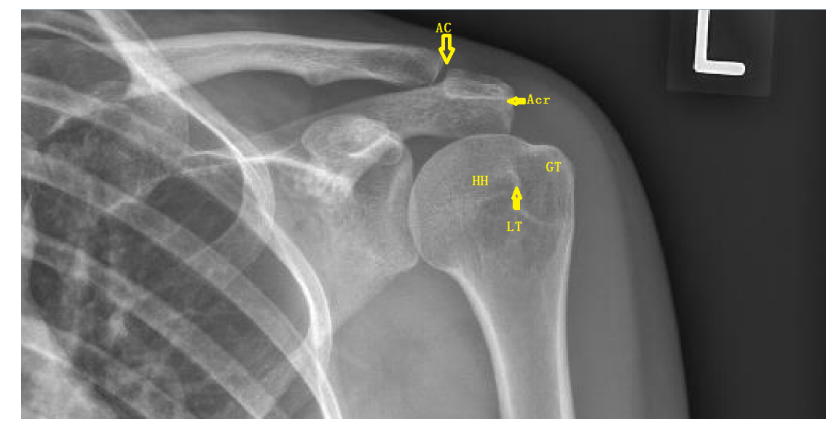

Figure 1 - Initial radiography of the left shoulder revealed no calcification in the lateral and posterior infraspinatus and the teres minor tendon. No stone or calcification show between Great tuberosity and the Acromion (Acr), the greater tuberosity (GT), the lesser tuberosity (LT),

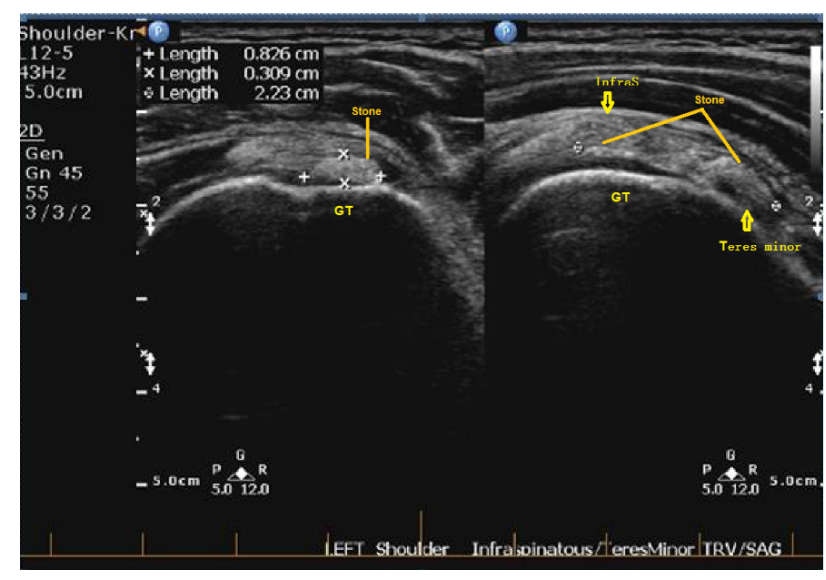

Figure 2 - MSK ultrasound revealed ill-defined calcifications in the infraspinatus and teres minor tendons junction, which is adjacent to the posterior humeral head that were consistent with calcific infraspinatus and teres minor tendinitis. Infraspinatus muscle tendon (InfraS ), Teres minor muscle tendon ( Teres minor), Great tuberosity (GT).

\section{Discussion}

Calcific tendinitis can affect any tendon in the body; however, with respect to pathology, calcific tendinitis around the rotator cuff has been the most frequently reported form of this condition. Among such cases, the most commonly affected tendon is the supraspinatus tendon. ${ }^{10,11,15,16}$ Typically, more than $80 \%$ of cases of total calcific tendinitis in the rotator cuff region occur in the supraspinatus tendon. These data are consistent with findings obtained by Louwerens et al. for both asymptomatic and symptomatic groups, which indicated that only $10 \%$ of all calcification deposits were found in the infraspinatus tendon, and no cases of calcific tendonitis in the teres minor region have been reported in the PubMed. ${ }^{4}$

In many cases the painfulness of calcific tendinitis is not produced by inherent aspects of the condition; instead, in most situations, painfulness is caused by tendon tears and bursal lesions correlated with calcific tendinitis. ${ }^{4,12}$ In most reported case studies, painfulness may also occur due to bone or cortical erosion. ${ }^{10,14,17}$ Another phenomenon that causes shoulder pain is sudden inflammation that produces an aggressive inflammatory reaction with inflammatory cell accumulation, excessive edema and increasing intratendineous pressure. ${ }^{6,15}$ This reaction leads to severe pain that certain researchers have attributed to secondary impingement resulting from increased tendon size or to the rupture of 
deposits into the subacromial space or the bursa. ${ }^{1,6}$ In the case described here, the patient experienced a sudden onset of extreme pain during his vacation. As ultrasound and X-ray results indicated, the patient had calcific tendinitis with inflammation around the calcifying area and hypoechoic foci in the tendons. Thus, the inflammation and tendon tears were thought to be the main cause of the patient's pain; typically, calcific tendinitis alone does not induce such severe pain.

Hypotheses regarding the relation between the size of the infraspinatus and teres minor tendon calcific tendinitis and the pain in the shoulder region have been discussed in many articles. ${ }^{1,4,18}$ In many cases, painfulness occurs suddenly, without harbingers. ${ }^{19}$ Thus, many research groups have argued that calcific tendinitis develops asymptomatically, but only to a certain degree. As the size of the calcific tendon grows, the pain increases. This pain does not increase over time as the calcification becomes larger; instead, calcification is thought to trigger pain when it reaches a certain threshold. ${ }^{19}$ In a study by Louwerens et al. of 1219 subjects with and without sub-acromial pain syndrome, the asymptomatic group have a median deposit length of $0.42 \mathrm{~cm}$; whereas the symptomatic group have a median deposit length of $1.16 \mathrm{~cm}$. This finding supports Bosworth's theory that large calcific deposits will always eventually result in a painful shoulder, although they may remain quiescent and symptomless for months or years. ${ }^{18}$ In the case described here, the calcific deposits were larger than $1.5 \mathrm{~cm}$, supporting Bosworth's theory that large calcific deposits result in pain. The patient's pain could also be explained by his inflammation and bursal lesion. Larger calcifying deposits have a higher chance of rupturing surrounding tissues, causing leakage of the bursa and inflammation. ${ }^{1}$

Many articles have compared different methods for finding and determining calcific tendinitis. $^{6,12,20}$ These articles suggest that ultrasound is relatively sensitive for detecting, identifying and localizing small calcific deposits or insertions between two tendons compared to X-ray and MRI. ${ }^{1,4,6,21}$ Ultrasound also provides an excellent means of evaluating the integrity of the rotator cuff tendons, bicep tendons, and subacromial bursa. However, ultrasound cannot be used to classify pathophysiological phases. ${ }^{6}$

\section{Conclusions}

This case report presented a rare case of calcific tendinitis of the rotator cuff that affects both the infraspinatus tendon and the teres minor tendon. Calcific tendinitis in this region is highly unusual and therefore necessary to report. Although no irregularities in pathology were noted for calcific tendinitis in this specific region, such rarely occurring forms of calcific ,tendinitis may suggest that all joint tendons could develop calcific tendinitis in the same manner. Further follow up on this case report is necessary to report the outcome for this specific case.

As we gain a better understanding of calcific tendinitis, there remain areas that merit additional discussion. We acknowledge that this disease's rate of occurrence is unevenly distributed in the population in correlation with factors such as age and sex. The lack of information on origin and natural history of calcific tendinitis pathology provides guidelines for further articles on diagnosis and research. In addition, few articles on prevalence and distribution of rotator cuff calcific tendinitis among large population samples have been published. Further evidence and more case reports on this topic are required to establish more details regarding this disorder.

\section{References}

1. Sansone V, Consoni O, Maiorano E, et al. Calcific tendinopathy of the rotator cuff: the correlation between pain and imaging features in symptomatic and asymptomatic female shoulders. Skeletal Radiol 2016;45:49.

2. McKendry RJ, Uhthoff HK, Sarkar K, et al. Calcifying tendinitis of the shoulder: prognostic value of clinical, histologic, and radiologic features in 57 surgically treated cases. J Rheumatol 1982;9(1):75.

3. Uhthoff HK, Loehr JW. Calcific tendinopathy of the rotator cuff: pathogenesis, diagnosis, and management. J Am Acad Orthop Surg. 1997;5:183.

4. Louwerens JK, Sierevelt IN, van Hove RP, et al. Prevalence of calcific deposits within the rotator cuff tendons in adults with and without subacromial pain syndrome: clinical and radiologic analysis of 1219 patients. J Shoulder Elbow Surg 2015;24:1588.

5. Chan R, Kim DH, Millett PJ, et al. Calcifying tendinitis of the rotator cuff with cortical bone erosion. Skeletal Radiol 2004;33:596.

6. EIShewy MT. Calcific tendinitis of the rotator cuff. World Journal of Orthopedics 2016;7:55.

7. di Cesare A, Giombini A, Dragoni S, et al. Calcific tendinopathy of the rotator cuff. conservative management with $434 \mathrm{Mhz}$ local microwave diathermy (hyperthermia): a case study. Disabil Rehabil 2008;30:1578.

8. de Conti G, Marchioro U, Dorigo A, et al. Percutaneous ultrasound-guided treatment of shoulder tendon 
calcifications: clinical and radiological follow-up at 6 months. $\mathrm{J}$ Ultrasound 2010;13:188.

9. Siegal DS, Wu JS, Newman JS, et al. Calcific tendinitis: a pictorial review. Can Assoc Radiol J 2009;60:263.

10. Porcellini G, Paladini P, Campi F, et al. Osteolytic lesion of greater tuberosity in calcific tendinitis of the shoulder. J Shoulder Elbow Surg 2009;18:210.

11. Farid N, Bruce D, Chung CB. Miscellaneous conditions of the shoulder: anatomical, clinical, and pictorial review emphasizing potential pitfalls in imaging diagnosis. Eur $\mathrm{J}$ Radiol 2008;68:88.

12. Sinués EM, Guillén APS, Marín ACV, et al. Case report: bilateral calcifying tendinitis of the long tendon of the biceps associated with a SLAP lesion. Reumatología Clínica 2010;6:145.

13. Aina R, Etiemme C, Bureau NJ, Aubin B, brassard P. Calcific shoulder treatment with modified U-S guided fine needle technique. Radiology. 2001;221:455.

14. Sola WC, Jr., Drake GN, Ramos $\mathrm{CH}$, et al. Calcific tendinitis of the rotator cuff associated with intraosseous loculation: two case reports. J Shoulder Elbow Surg 2009;18:e6.

15. Martin S, Rapariz JM. Intraosseous calcium migration in calcifying tendinitis: a rare cause of single sclerotic injury in the humeral head (2010: 2b). Eur Radiol 2010;20:1284.

16. Rahu M, Kolts I, Poldoja E, et al. Rotator cuff tendon connections with the rotator cable. Knee Surg Sports Traumatol Arthrosc 2016. doi: 10.1007/s00167-016-4148-4.

17. Durr HR, Lienemann A, Silbernagl H, et al. Case report: acute calcific tendinitis of the pectoralis major insertion associated with cortical bone erosion. Eur Radiol 1997;7:1215.

18. Bosworth BM. Calcium deposits in the shoulder and subacromial bursitis: a survey of 12,122 shoulders. JAMA 1941;116:2477.

19. Diehl P, Gerdesmeyer L, Gollwitzer $\mathrm{H}$, et al. Calcific tendinitis of the shoulder. Orthopade 2011;40:733.

20. Zubler C, Mengiardi B, Schmid MR, et al. MR arthrography in calcific tendinitis of the shoulder: diagnostic performance and pitfalls. Eur Radiol 2007;17:1603.

21. Wallny TA, Theuerkauf I, Schild RL, et al. Basic science: original paper, the three-dimensional ultrasound evaluation of the rotator cuff--an experimental study. European Journal of Ultrasound 2000;11:135. 